\title{
Principes physiques des lasers à semiconducteurs
}

\author{
V. Berger \\ Thales Research and Technology, Domaine de \\ Corbeville, 91400 ORSAY, FRANCE \\ vincent.berger@thalesgroup.com
}

Résumé. Les principes physiques des lasers à semiconducteurs sont passés en revue. Après quelques rappels fondamentaux de physique des solides concernant les états électroniques dans les semiconducteurs et les concepts de fonction de Bloch ou de bande interdite, la manière dont un semiconducteur peut émettre de la lumière sera examinée. La jonction $p$-n est le dispositif de base permettant d'obtenir l'inversion de population nécessaire au gain d'émission stimulée. Nous exposons les principes et les équations fondamentales régissant le transport électronique dans ces structures. L'équation de seuil des lasers à semiconducteurs est ensuite déterminée et les différentes améliorations par rapport à la jonction p$\mathrm{n}$ de base (le laser à double hétérostructure, le laser à puits quantique) sont exposées dans une perspective historique. Enfin, la diversité des diodes lasers est illustrée par quelques variations : lasers de puissance, lasers à cavité verticale émettant par la surface. La synthèse est suivie d'une tentative de mise en perspective des avancées futures des lasers à semiconducteurs.

\section{INTRODUCTION}

Cet article a l'ambition de décrire simplement les principes physiques généraux des lasers à semiconducteurs. Ces dispositifs ont aujourd'hui une importance énorme dans notre civilisation : télécommunications par fibre optique (internet, téléphone, télévision...), stockage de l'information dans les disques optiques (CD ou DVD pour la musique comme pour l'informatique), photocopie ou impression laser, applications médicales et industrielles... Tout cela représente aujourd'hui $70 \%$ du marché total des lasers, soit environ 3 milliards de \$ en l'an 1999.

Mots-clés : Semiconducteurs, lasers à semiconducteurs, diodes lasers, jonction p-n. 


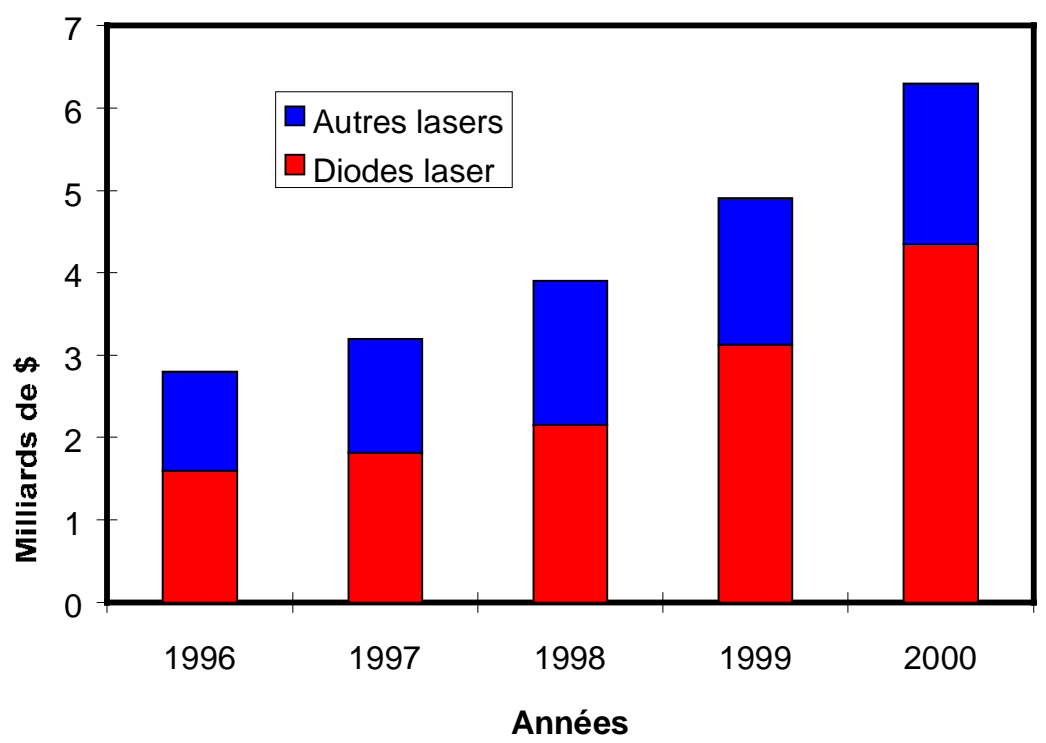

FIG. 1. Le marché des lasers.

La croissance d'un tel marché a eu lieu en moins d'une vingtaine d'années, et a naturellement été accompagnée d'une recherche phénoménale, aussi bien fondamentale qu'appliquée, pour comprendre et améliorer le fonctionnement des dispositifs, et également pour inventer des versions différentes des diodes lasers, avec leurs spécificités. Aujourd'hui les lasers à semiconducteurs sont très loin d'avoir épuisé leur potentiel d'innovation et d'avoir démontré toutes leurs possibilités. Porté par un marché des télécommunications dont les besoins sont immenses et encore très loin d'être assouvis, le domaine des diodes laser est également appelé à conquérir de nouveaux marchés grâce à une extension de ses possibilités. Citons par exemple les nouvelles gammes spectrales nouvellement proposées par les lasers à semiconducteurs, des deux côtés du spectre visible : l'UV ou le bleu d'une part, avec des applications dans le stockage d'information de haute densité ou dans l'imagerie laser, mais aussi l'infrarouge moyen d'autre part, et ses applications dans le domaine de la détection de gaz ou dans le domaine de la défense. Rentrer dans le détail des différentes innovations technologiques qui ont enrichi le domaine des lasers à semiconducteurs nécessiterait l'espace d'une encyclopédie. Dans le cadre de ce cours, nous nous limiterons aux idées génériques permettant de comprendre les bases du fonctionnement de ces composants.

\section{LES ÉTATS ÉLECTRONIQUES DANS UN SEMICONDUCTEUR}

Cette première partie rappelle simplement la description des états électroniques dans un cristal semiconducteur. Ces notions sont naturellement indispensables pour comprendre comment un cristal peut émettre de la lumière. Le physicien qui cherche à 
décrire les états possibles d'un électron dans un cristal doit résoudre l'équation de Schrödinger en présence du potentiel cristallin c'est-à-dire le potentiel constitué par la totalité des noyaux atomiques rangés de manière périodique et formant le cristal. Le problème est naturellement très complexe : à ce potentiel « fixe » des noyaux atomiques du cristal s'ajoute le potentiel provenant des autres électrons présents dans le cristal. Pour connaître ce potentiel d'interaction coulombienne, il faudrait cependant déjà connaître les états électroniques, ce qui est précisément ce que l'on cherche! La résolution du problème nécessite donc la considération de l'équation de Schrödinger et de celle de Poisson de manière couplée, et la difficulté est considérable. Heureusement, quelques idées simples amènent néanmoins à l'essentiel des notions qualitatives nécessaires pour comprendre ce qui se passe.

Une première approche pour appréhender les états électroniques dans un cristal consiste à considérer celui-ci comme une grosse molécule. Nous appellerons cette approche la méthode du chimiste. Le problème de la molécule d'hydrogène est un cas d'école classique : la résolution de l'équation de Schrödinger pour le double puits quantique que représentent les deux noyaux montre que la levée de dégénérescence entre les deux niveaux d'énergie sur les atomes d'hydrogène considérés séparément mène à deux niveaux couplés, l'un dit liant, de plus basse énergie, et l'autre, dit anti-liant, de plus haute énergie. De la même manière, si on augmente le nombre $N$ d'atomes couplés dans une molécule (pour atteindre la molécule gigantesque que constitue notre cristal), on comprend intuitivement que le couplage entre tous les niveaux individuels sur chaque atome va former un ensemble de $N$ états (une «bande d'énergie»). La bande d'énergie électronique dans un cristal résulte donc de l'hybridation des niveaux individuels des atomes qui composent le cristal. L'ensemble des bandes d'énergie du solide ne recouvre pas forcément tout le spectre des énergies possibles, et il peut rester entre ces bandes d'énergie permise des bandes d'énergie interdite. Cela signifie simplement qu'un électron ne peut pas avoir n'importe quelle énergie dans un cristal, il occupe nécessairement un état à l'intérieur d'une bande permise. Ce résultat n'est qu'un élargissement à l'état solide du résultat bien connu de la mécanique quantique appliquée à l'atome, qui dit qu'un électron dans un atome ne peut pas avoir n'importe quelle énergie : il occupe nécessairement un niveau d'énergie précis, donné par l'équation de Schrödinger.

Une deuxième approche, plus abstraite, mène au même résultat. C'est la méthode du mathématicien. Elle utilise le théorème de Bloch, qui stipule que, dans un potentiel périodique, toutes les solutions de l'équation de Schrödinger sont des fonctions dites de Bloch, c'est-à-dire qu'il existe un vecteur $\mathbf{k}$ permettant d'écrire :

$$
\Psi_{\mathbf{k}}(\mathbf{r})=u_{\mathbf{k}}(\mathbf{r}) \cdot \exp (i \mathbf{k} . \mathbf{r})
$$

où $u_{\mathbf{k}}(\mathbf{r})$ est une fonction périodique avec les mêmes périodes que le potentiel. Les fonctions d'ondes des électrons dans un cristal parfait (périodique, infini, sans défaut ...) sont donc simplement le produit entre une onde plane et une fonction périodique. Au fond, ce sont des ondes planes (témoignant de la délocalisation et de la liberté des électrons) qui se construisent sur le paysage cristallin. Ce qui est intéressant avec cette écriture, c'est que l'on a maintenant indexé toute fonction d'onde par un vecteur d'onde $\mathbf{k}$. On peut donc considérer le problème $\mathbf{k}$ par $\mathbf{k}$ et chercher à résoudre, pour un $\mathbf{k}$ donné, l'équation de Schrödinger. On va ainsi trouver, pour ce 


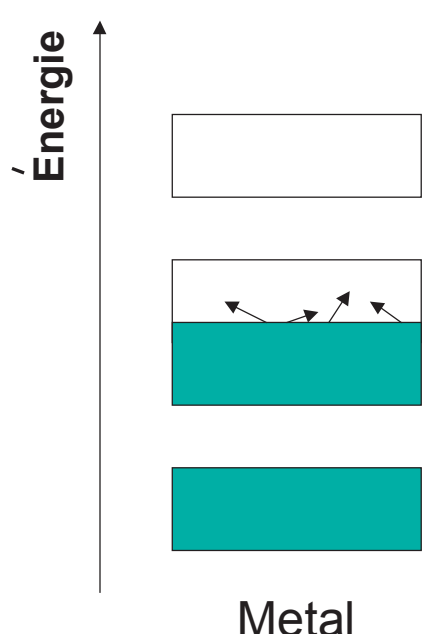

Metal

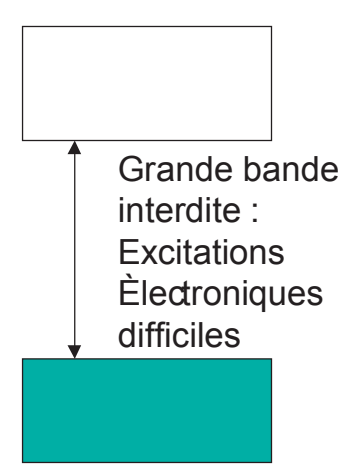

Isolant

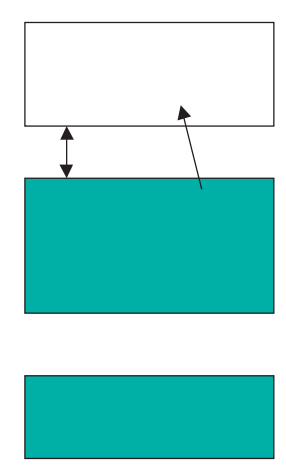

Semi-conducteur

FIG. 2. Métal, isolant, semi-conducteur.

$\mathbf{k}$ donné, une équation qui porte sur les fonctions périodiques $u_{\mathbf{k}}(\mathbf{r})$. C'est là que le problème a été simplifié d'une manière monumentale. Alors que l'équation de Schrödinger cherche des solutions dans tout l'espace des fonctions a priori, le théorème de Bloch permet de réduire le problème en tirant profit de la périodicité du potentiel : pour chaque vecteur $\mathbf{k}$, on cherche maintenant des solutions dans l'espace des fonctions périodiques. Or cet espace est d'une dimension dénombrable (puisqu'on peut décomposer une fonction périodique en série de Fourier à l'aide d'un ensemble dénombrable de coefficients) ; l'ensemble des solutions (et des énergies possibles) est donc également dénombrable, pour chaque $\mathbf{k}$. Par continuité entre les différents $\mathbf{k}$, on construit ainsi un ensemble dénombrable de bandes d'énergie que l'on représente sous la forme d'une relation de dispersion $E(\mathbf{k})$ appelé aussi diagramme ou structure de bande. On retrouve, par ces arguments certes un peu plus abstraits, le concept de bandes d'énergie permise et interdite.

Notons que l'on retrouve dans la partie périodique des fonctions de Bloch une réminiscence des orbitales atomiques de départ. En effet, la méthode du chimiste nous a enseigné que ces états électroniques sont obtenus par hybridation des orbitales atomiques. Leur trace est donc conservée dans la partie périodique de la fonction de Bloch, en particulier avec ses propriétés de symétrie.

Le physicien doit garder à l'esprit ces deux méthodes, celle du mathématicien comme celle du chimiste. Chacune permet, selon le problème traité ou l'argument recherché, d'apporter un éclairage vraiment différent, en particulier parce que l'une s'intéresse à l'espace réel, en partant du potentiel local; l'autre évolue d'emblée dans l'espace réciproque, en considérant le potentiel global. Mais il ne serait pas raisonnable de passer plus de temps ici sur ces considérations, le lecteur est invité pour approfondir ces notions à consulter l'excellent ouvrage de Sutton, Electronic Structures of Materials (Oxford). 
De l'existence des bandes d'énergie électroniques va découler immédiatement des propriétés de transport essentielles des solides. Comme le montre la figure 2, la possibilité d'appliquer à un électron de la mer de Fermi une excitation élémentaire dépend de la position du niveau de Fermi dans ces bandes. Si le niveau de Fermi est situé à l'intérieur d'une bande d'énergie, le nombre d'états immédiatement accessibles à un électron de la mer de Fermi est grand et il sera facile d'exciter un tel électron. On a alors un métal, matériau conduisant bien l'électricité et la chaleur. En revanche, il sera très coûteux en énergie (et donc très difficile) d'exciter le moindre électron si le nombre d'électrons dans le solide est tel qu'une bande est complètement remplie et la suivante complètement vide. Une telle coïncidence n'est en réalité pas complètement un hasard car on a au total N.p électrons ( $p$ nombre d'atomes) et $p$ états dans chaque bande obtenu par hybridation des $p$ niveaux. Une telle coïncidence entre le dernier état occupé et le haut d'une bande peut donc arriver fréquemment : on a alors un isolant.

Le cas du semiconducteur est intermédiaire : le semiconducteur est un isolant de faible bande interdite. La dernière bande pleine est appelée bande de valence, la première bande inoccupée est appelée bande de conduction. Une excitation électronique est beaucoup plus difficile que dans le cas d'un métal, mais reste possible, car la largeur de la bande interdite (ou gap) est faible. On peut en particulier rendre un semiconducteur vraiment conducteur en plaçant quelques électrons en bande de conduction (d'où ce nom...) ou, de manière équivalente, en enlevant quelques électrons de la bande de valence, ce qui libère des états vides pour le transport dans cette bande. Ces absences d'électrons dans la bande de valence sont appelées des trous et sont finalement assimilées à des particules de charge positive qui transportent le courant. La figure 3 montre différents moyens d'arriver à cette population conductrice : par la température (dès que l'énergie thermique $k T$ devient non complètement négligeable devant l'énergie de bande interdite, des paires électron-trou sont créées) ou par dopage. Le dopage est la substitution d'atomes du cristal semiconducteur par des atomes contenant un nombre d'électrons de valence immédiatement supérieur ou inférieur. Dans le premier cas, l'atome (appelé alors atome donneur) apporte un électron de plus que nécessaire pour réaliser les liaisons cristallines normales et remplir les bandes d'énergie du semiconducteur. Cet électron supplémentaire finit donc en bande de conduction, comme surplus. On parle alors de semiconducteur dopé $n$, et le niveau de Fermi dans ce cas est situé tout près ou dans la bande de conduction. Dans le second cas où l'on place dans le cristal des atomes (appelés cette fois accepteurs) auxquels il manque un électron de valence, il va cette fois manquer d'électrons pour finir de remplir la bande de valence (on parle alors de dopage $p$ ); le niveau de Fermi est situé en bande de valence et on a alors une conduction par trous possible. L'ensemble de ces notions de base sur les semiconducteurs est ici résumé de manière très succincte et le lecteur se reportera aux très nombreux ouvrages sur le sujet pour une description plus complète. Citons par exemple l'ouvrage de Sze, Physics of Semiconductor Devices (John Wiley \& Sons), dans lequel le fonctionnement de la jonction $p-n$ est également traité de manière exhaustive. 


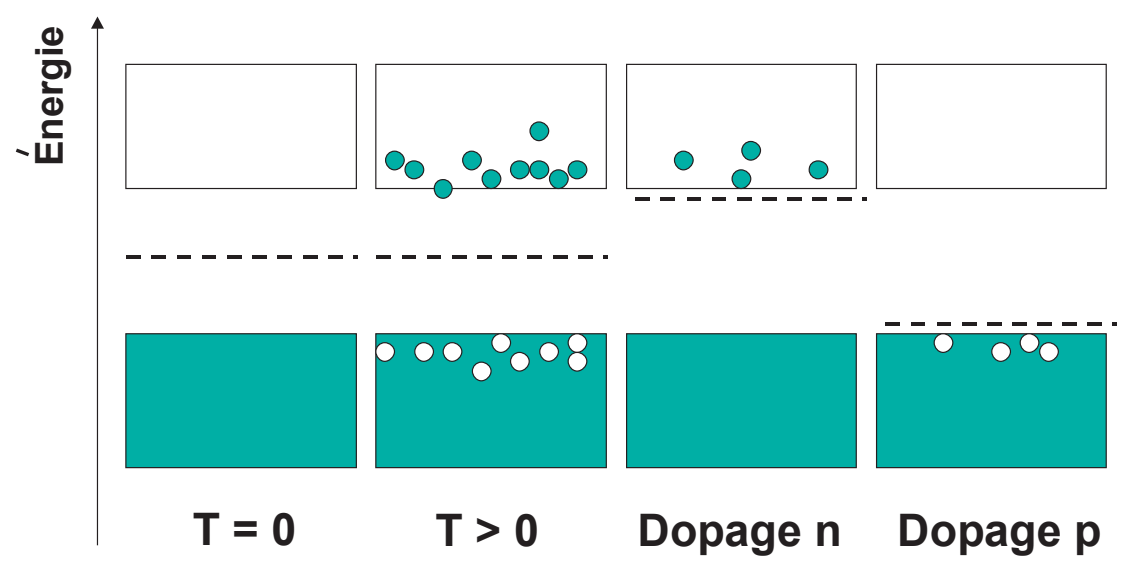

Fig. 3. Du semi-conducteur au conducteur. La ligne pointillée représente le niveau de Fermi.

\section{L'ÉMISSION DE LUMIÈRE PAR UN SEMICONDUCTEUR}

Il reste un point important à signaler pour finir cette description des états électroniques dans les semiconducteurs : la dynamique des porteurs dans ces bandes. La figure 4 représente le diagramme de dispersion $E(\mathbf{k})$ d'un semiconducteur tel que le GaAs. Toutes les bandes ne sont naturellement pas représentées ici; seuls le haut de la bande de valence et le bas de la bande de conduction sont reportés car c'est ici que se situe tout l'intérêt : là où les excitations électroniques peuvent avoir lieu. Imaginons qu'à la suite de l'absorption de l'énergie d'un photon, un électron soit promu de la bande de valence vers la bande de conduction. Cet électron va redescendre en bas de la bande de conduction à la recherche des états de plus basse énergie, en un temps sub-picoseconde. Cette rapidité extrême est due à la densité continue d'états disponibles le long de cette relaxation qui s'effectue donc très efficacement via des collisions avec des phonons ou d'autres électrons. De même, le trou créé va se relaxer en haut de la bande de valence (le trou est une absence d'électron, ce qui signifie recherche de l'énergie la plus haute, comme une bulle de champagne qui remonte à la surface), en un temps également sub-picoseconde. La relaxation finale aura lieu quand l'électron aura réintégré la bande de valence en se recombinant avec le trou. Mais le saut en énergie nécessaire est alors beaucoup plus grand et ne peut pas se faire avec une simple interaction avec un phonon par exemple. C'est pourquoi ce temps de relaxation inter-bande est beaucoup plus long que le temps de relaxation intra-bande. Nous verrons dans la section suivante que cette recombinaison inter-bande peut se faire via l'émission d'un photon, mais le temps mis en jeu, de l'ordre de la nanoseconde, est supérieur de plus de trois ordres de grandeur au temps de relaxation intra-bande. Ceci est un point à retenir, qui justifie le concept de quasi-niveau de Fermi : si on place une population d'électron (resp. de trous) dans la bande de conduction (resp. de valence), la population d'électrons (resp. de trous) trouve rapidement (en un temps sub-picoseconde) un équilibre thermodynamique 


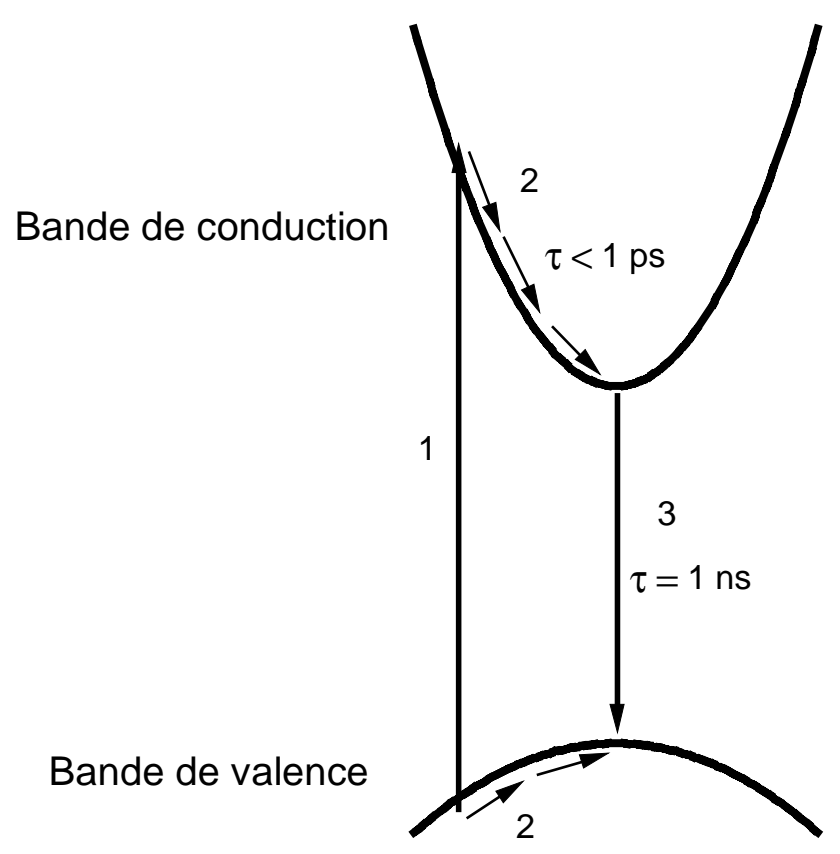

FIG. 4. - Diagramme de dispersion $E(\mathbf{k})$ dans un semiconducteur tel que GaAs. En ordonnée, l'énergie et en abscisse le vecteur d'onde sont sous-entendus. Les flèches représentent schématiquement les différents processus lors d'une expérience de photoluminescence : absorption (1), relaxation intrabande (nonradiative, 2) puis inter-bande (luminescence, 3).

dans la bande de conduction (resp. de valence). Ces deux équilibres locaux durent le temps (long) précédant la relaxation inter-bande et peuvent donc se décrire séparément avec un niveau de Fermi en bande de conduction et un autre en bande de valence. On les appelle quasi-niveaux de Fermi car il ne saurait y avoir deux niveaux de Fermi au plan strict du terme. Les quasi-niveaux de Fermi décrivent deux situations d'équilibre local (une en bande de conduction, l'autre en bande de valence), l'équilibre global entre ces deux bandes n'étant pas encore réalisé.

La description de l'émission d'un photon lors de la relaxation d'un électron d'un état excité d'un atome vers un état de plus faible énergie met en jeu un élément de matrice entre les fonctions d'onde finales, initiales, et le hamiltonien d'interaction (voir Fig. 5). Il en est de même pour l'absorption d'un photon par un électron, processus inverse décrit par le même élément de matrice. Cette démarche, appliquée aux fonctions de Bloch dans un solide, mène à un résultat aux conséquences très importantes : comme on le voit sur la figure 5, la décomposition de l'élément de matrice fait apparaître une intégrale comprenant un terme $\exp \left(i\left(\mathbf{k}_{f}-\mathbf{k}_{i}+\mathbf{k}_{\text {photon }}\right) \cdot \mathbf{r}\right)$ et un terme produit des parties périodiques des fonctions de Bloch $u_{\mathbf{k} f}(\mathbf{r}) u_{\mathbf{k} i}(\mathbf{r})$, où $i$ et $f$ désignent les états initial et final de l'électron. Ce second terme ressemble en quelque sorte à un élément de matrice atomique, puisque nous avons dit que les parties périodiques des fonctions de Bloch ont une allure de fonction d'onde 


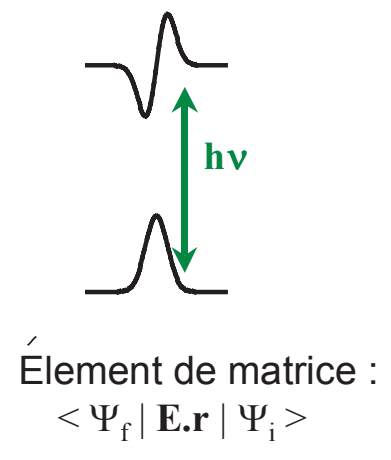

Absorption "verticale" (conservation du vecteur $\mathbf{k}$ )

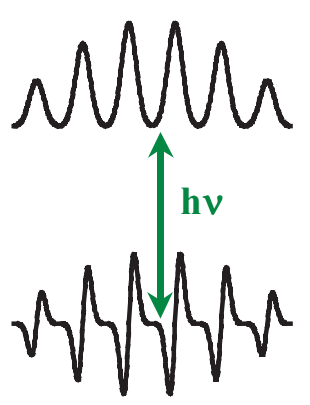

$$
<\Psi_{\mathrm{f}}|\mathbf{E} . \mathbf{r}| \Psi_{\mathrm{i}}>=
$$$$
\int \exp \left[i\left(\mathbf{k}_{\mathrm{f}}-\mathbf{k}_{\mathrm{i}}+\mathbf{k}_{\text {photon }}\right) \cdot \mathbf{r}\right] \cdot \mathrm{u}_{\mathrm{kf}}(\mathbf{r}) \mathrm{u}_{\mathrm{ki}}(\mathbf{r}) \ldots
$$

$\downarrow$

Élement de matrice "atome-like"

FIG. 5. Transition optique dans un atome (à gauche) et dans un semiconducteur (à droite).

atomique. Il peut contenir en particulier des règles de sélection sur la polarisation des photons émis. Le premier terme exponentiel, quant à lui, annule l'élément de matrice dès que la somme $\mathbf{k}_{f}-\mathbf{k}_{i}+\mathbf{k}_{\text {photon }}$ est non nulle. On a donc nécessairement dans toute transition optique conservation du vecteur d'onde, qui apparait chaque fois qu'une invariance par translation a lieu, comme c'est le cas ici. Le vecteur d'onde du photon pouvant la plupart du temps être négligé dans l'échelle des vecteurs d'ondes électroniques d'intérêt, ceci revient à dire que $\mathbf{k}_{f} \approx \mathbf{k}_{i}$; ou encore que l'on a une transition «verticale» dans l'espace des $\mathbf{k}$, comme cela est dessiné sur la figure 4 . Cette remarque a des conséquences très lourdes. En particulier, dans un semiconducteur comme le Silicium, où la base de la bande de conduction et le haut de la bande de valence ne se situent pas au même vecteur $\mathbf{k}$ (on parle de semiconducteur à gap « indirect »), si l'on créé une population d'électrons et de trous, ceux-ci sont relaxés en un temps sub-picoseconde en deux points différents de l'espace des $\mathbf{k}$. Toute relaxation radiative est alors très difficile. Un semiconducteur à bande interdite indirecte est donc un très mauvais émetteur de lumière et c'est pourquoi le Silicium qui a eu tant de succès en microélectronique n'est pas un bon matériau pour l'optoélectronique. En revanche, un matériau à bande directe comme le GaAs, pour lequel électrons et trous se retrouvent sur les maxima de leurs bandes respectives qui sont tous les deux au point $\mathbf{k}=0$ (voir Fig. 4), est un très bon émetteur de lumière. En résumé :

«God created Si for microelectronics, and GaAs for optoelectronics. And Carbon for life»

R. Suris (communication privée).

Le lecteur désirant approfondir ses connaissances sur les processus optiques dans les semiconducteurs est invité à consulter par exemple l'ouvrage de Pankove, Optical Processes in Semiconductors (Dover Publications). 


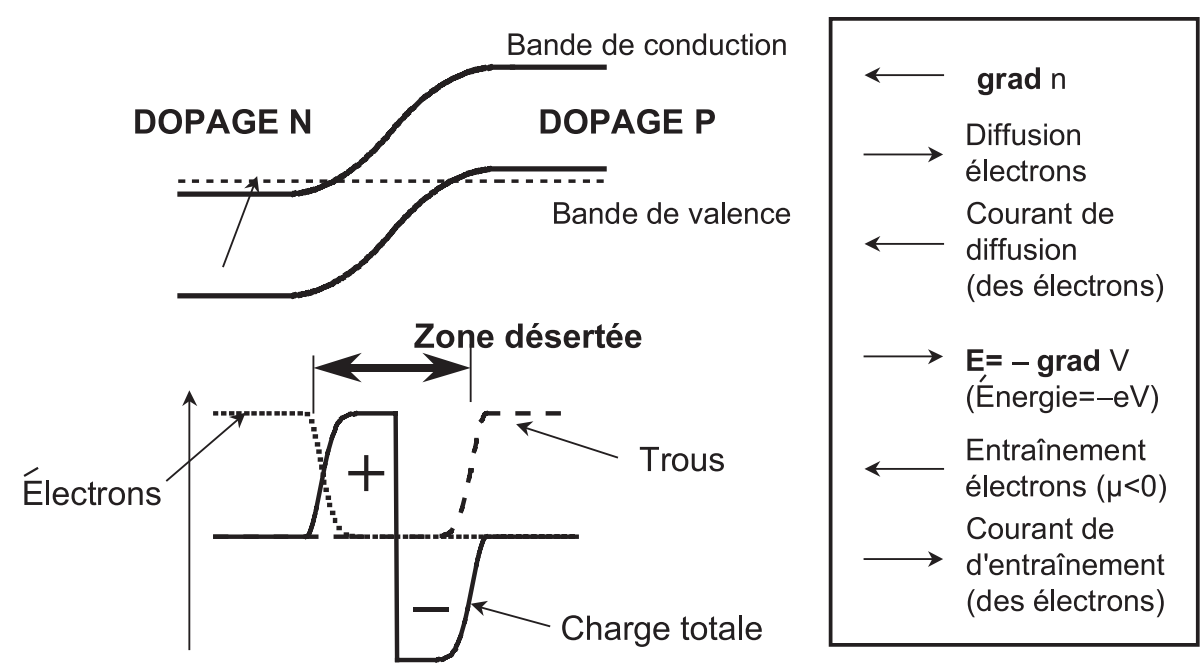

FIg. 6. - Jonction $p$ - $n$ à l'équilibre thermodynamique. La charge totale présente dans la zone de charge d'espace (qui est responsable du champ interne) vient des charges fixes (atomes dopants).

Nous savons maintenant comment un semiconducteur peut absorber ou émettre de la lumière : par création d'une paire électron-trou, ou par recombinaison d'un électron avec un trou, respectivement. Pour un semiconducteur à l'équilibre thermodynamique, il y a énormément plus d'électrons en bande de valence qu'en bande de conduction et donc, globalement, le matériau absorbe les photons d'énergie supérieure à l'énergie de bande interdite. Pour réaliser un laser, il faut maintenant amener suffisamment d'électrons et de trous pour obtenir l'inversion de population. Ce fantastique déséquilibre thermodynamique sera obtenu dans un dispositif appelé jonction $p-n$.

\section{LA JONCTION $p$ - $n$ ET L'INVERSION DE POPULATION}

Imaginons un semiconducteur dopé $n$, obtenu en incorporant des atomes donneurs lors de sa croissance cristalline. Puis, à un certain point de cette croissance, imaginons que l'on arrête d'incorporer des atomes donneurs, pour les remplacer au contraire par des atomes accepteurs. Le résultat est alors un semiconducteur dopé $n$ dans une première partie, puis dopé $p$ dans une seconde partie. Ce type de structure appelée «jonction $\boldsymbol{p}-\boldsymbol{n}$ » constitue la base des diodes lasers. Étudions d'abord la jonction $p-n$ à l'équilibre, c'est-à-dire sans qu'aucun champ électrique ne soit appliqué.

Une remarque simple permet de comprendre tout de suite l'allure du potentiel de la jonction $p-n$, représenté sur la figure 6 : à l'équilibre thermodynamique, il n'y a par définition qu'un seul niveau de Fermi. Étant donné que, loin de la jonction côté $p$, le niveau de Fermi est situé dans ou est proche de la bande de valence, et que de 
manière équivalente, loin du côté $n$, le niveau de Fermi est situé dans la bande de conduction, il y a nécessairement au niveau de l'interface $p$ - $n$ un champ électrique induit qui permet de raccorder l'ensemble par continuité. Ce champ représente une barrière qui empêche les électrons (resp. les trous) de diffuser dans la zone $p$ (resp. $n$ ). Après cette remarque de bon sens, regardons tout cela d'un peu plus près.

Nous nous trouvons en présence d'une concentration inhomogène en électrons et en trous : nous sommes a priori en présence de beaucoup d'électrons dans la zone $n$ et de beaucoup de trous dans la zone $p$. Cette inhomogénéité a pour conséquence l'établissement d'un courant de diffusion, selon la loi de Fick :

$$
\begin{array}{ll}
j_{n}(x)=-D \nabla n(x) & \text { (densité de courant de particules) } \\
j(x)=e D \nabla n(x) & \text { (densité de courant électrique). }
\end{array}
$$

Les grandeurs ici sont scalaires dans la mesure où le problème est à une dimension. À l'équilibre thermodynamique, le courant total d'électrons est pourtant nécessairement nul. Il existe donc un courant en sens inverse qui compense cette diffusion d'électrons vers la zone $p$. Un électron qui quitte la zone $n$ pour diffuser dans la zone $p$ laissant derrière lui une charge fixe positive (le noyau de l'atome donneur), il en résulte une force coulombienne de rappel. Cette force correspond précisément au champ électrique induit dans la structure que nous avons déjà évoquée, et ce champ électrique est tel que le courant d'électrons lié à ce champ interne compense exactement le courant de diffusion à l'équilibre. En d'autres termes, les électrons diffusent jusqu'à ce que le champ de charge d'espace créé par les atomes donneurs soit devenu tellement important qu'il empêche les électrons de diffuser plus encore par l'établissement d'une barrière de potentiel.

L'équilibre entre courant de diffusion et courant d'entraînement s'écrit :

$$
n(x) e \mu E(x)=e D \nabla n(x) .
$$

On a évidemment de manière symétrique le même type d'équation pour la concentration de trous en bande de valence. Les trous et les électrons ne coexistent pas à l'interface (sinon ils se recombineraient entre eux tout simplement); il existe donc une zone désertée de charges libres, de charge positive dans la zone $n$ (charge des atomes donneurs restant) et négative dans la zone $p$ (charge des atomes accepteurs). Le champ de charge d'espace résulte précisément de ces charges positives et négatives qui se font face de part et d'autre de la jonction.

L'équation d'équilibre (1) peut être retrouvée par un autre raisonnement. L'état d'équilibre thermodynamique implique que l'on peut décrire la population d'électrons dans la bande de conduction par la fonction de Fermi-Dirac. La densité d'électrons en fonction de l'abscisse s'écrit donc comme la somme, pour toutes les énergies, de la densité d'états $D_{\mathrm{c}}(E)$ multipliée par le taux d'occupation :

$$
n(x)=\int_{E_{\mathrm{c}}(x)}^{+\infty} \exp \left(\frac{E_{F}-E}{k T}\right) \times D_{\mathrm{c}}(E) \mathrm{d} E .
$$

Ici, la fonction d'occupation de Fermi-Dirac a été remplacée par l'approximation exponentielle de Maxwell-Boltzman, ce qui est une approximation acceptable pour un dopage raisonnable. 
La densité d'états évolue comme la racine de l'énergie d'un électron en bande de conduction $\left(E-E_{\mathrm{c}}\right)$, et le changement de variable $E-E_{\mathrm{c}}(x)$ montre que :

$$
n(x) \propto \exp \left(\frac{E_{F}-E_{\mathrm{c}}(x)}{k T}\right)=\exp \left(\frac{e V(x)}{k T}\right),
$$

où $V(x)$ est le potentiel en bande de conduction. La différentiation de cette équation mène à :

$$
\frac{\nabla n}{n}=\frac{e}{k T} \nabla V=\frac{-e}{k T} E .
$$

On retrouve facilement la relation (1) à l'aide de la relation d'Einstein :

$$
\frac{D}{\mu}=\frac{-k T}{e} .
$$

Sur la figure 6 sont représentées schématiquement la structure de bande de la jonction $p$ - $n$ avec le champ interne résultant des charges fixes et les densités d'électrons et de trous qui sont très faibles dans la zone désertée, de part et d'autre de la jonction. Les sens des différents courants qui se compensent sont également indiqués pour les électrons.

Quittons maintenant l'équilibre thermodynamique, en appliquant une tension positive $V$ (c'est-à-dire un potentiel positif sur la zone $p$ ). Toute la tension s'applique dans la zone désertée, puisqu'elle est en série avec les zones peuplées $n$ et $p$, beaucoup moins résistives. On touche là à la grande caractéristique des matériaux semiconducteurs, par rapport aux métaux ou aux isolants : on sait moduler la conductivité du matériau en ajoutant ou en retirant ici ou là les porteurs du courant électrique. Ce principe est à la base du fonctionnement du transistor et donc de la microélectronique : à l'aide d'une tension, on peut rendre un canal conducteur ou non en le peuplant ou non d'électrons de conduction. C'est le même principe qui est ici à la base de la diode laser : on sait faire varier, dans le même cristal, sur une épaisseur extrêmement faible, la conductivité. Dans la jonction $p$ - $n$, on a créé une zone désertée, donc très résistive, d'épaisseur typique $1 \mu \mathrm{m}$. Toute tension appliquée se concentre dans cette très petite partie du cristal et c'est à cause de cette dimension très réduite sur laquelle on peut créer un déséquilibre thermodynamique très intense que l'on peut atteindre l'inversion de population. La tension appliquée entraîne un abaissement du champ interne : la barrière de diffusion est abaissée d'une énergie $\mathrm{eV}$. En conséquence, l'équilibre précédent, formalisé par l'équation (1), est rompu et la diffusion d'électrons et de trous dans la zone désertée l'emporte. On se retrouve avec des électrons et des trous au même endroit dans la zone centrale : la recombinaison radiative est donc possible. Signalons même que tout passage du courant implique une recombinaison : en effet dans la zone $n$ le courant est transporté par des électrons; dans la zone $p$ le courant est transporté par des trous. Pour qu'un courant passe à travers l'ensemble du dispositif, il est donc nécessaire qu'une recombinaison ait lieu à l'interface. Cela ne signifie pas que tout passage d'électron implique l'émission d'un photon. Il existe aussi des recombinaisons non radiatives impliquant par exemple des phonons avec des niveaux de défauts au centre de la bande interdite. 


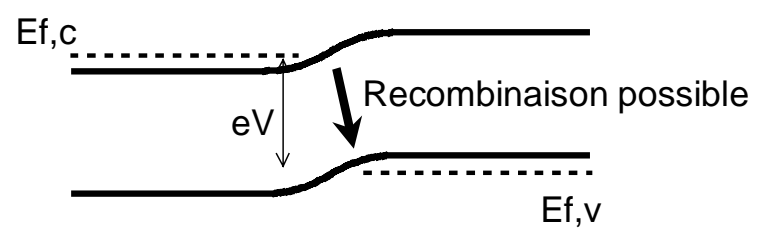

FIG. 7. La jonction $p$ - $n$ hors équilibre.

On peut ainsi définir un rendement quantique interne $\eta_{i}$ comme le rapport entre le nombre d'électrons recombinés radiativement sur le nombre d'électrons total passés dans la jonction. Sur la figure 7, on notera les deux quasi-niveaux de Fermi en pointillés en bande de valence et de conduction : ceux-ci ont un sens dans la mesure où, comme on l'a discuté plus haut, l'établissement d'un quasi-équilibre dans chacune des deux bandes est immédiat par rapport à la redescente des électrons de la bande de conduction à la bande de valence ; qui constitue réellement l'événement bloquant (le goulot d'étranglement) du transport à travers la structure.

Nous venons en réalité d'expliquer le fonctionnement de la diode émettrice de lumière (DEL ou LED en anglais), telle qu'on peut la voir par exemple sur les panneaux à message variable des autoroutes, ou telle qu'on ne peut pas la voir mais seulement la deviner dans une télécommande de téléviseur. La largeur spectrale d'une diode émettrice de lumière est assez élevée (environ 1,5 kT, soit $40 \mathrm{meV}$ ). Les pertes non radiatives, qui font chuter le rendement quantique, sont à éviter en améliorant la qualité du matériau. Un problème important concernant les diodes émettrices de lumière est l'extraction de la lumière de l'intérieur du matériau vers l'extérieur. En effet, une fois un photon émis, il n'est pas du tout sûr qu'il sortira du semiconducteur : en raison du fort indice de celui-ci, l'angle de réflexion totale interne est faible : il est en gros égal à $17^{\circ}$ pour GaAs, ce qui signifie que seulement $2 \%$ de l'angle solide total permet aux photons de sortir du semiconducteur sur une face. En conséquence, très nombreux sont les photons qui sont réfléchis à l'intérieur et finalement réabsorbés! L'énorme enjeu commercial autour des LEDs motive pour cela aujourd'hui toute une communauté à la recherche de la structure optique idéale pour sortir la lumière d'une diode : surfaces corruguées, miroirs anti-reflets, microcavités, pyramides, structures à bande interdite photonique... Le problème n'est pas simple du tout.

À ce stade, nous savons comment émettre de la lumière à partir d'une structure semi conductrice sous tension. Pour réaliser un laser, il faut aller plus loin en régime d'inversion de population. Il faut également mettre le milieu amplificateur ainsi obtenu dans une cavité.

\section{CONDITION DE SEUIL D’UN LASER À SEMICONDUCTEUR}

En augmentant la tension appliquée à la jonction $p$ - $n$ et donc le courant qui la traverse, on augmente la densité d'électrons et de trous présente dans la zone de charge d'espace. Si l'on note $\tau$ le temps typique qu'il faut aux électrons avant de se recombiner (de l'ordre de la nanoseconde) et si l'on affecte à la zone de recombinaison la 
largeur typique $d$, la densité volumique d'électrons (et de trous) dans cette zone est simplement :

$$
n=\frac{j \tau}{q d}
$$

Cette équation montre qu'il suffit d'augmenter le courant injecté dans la diode pour augmenter la population électronique et donc atteindre, à un certain point, l'inversion de population. Pour un courant faible, on a de l'absorption, puisque la bande de valence est pleine d'électrons et la bande de conduction vide, dans la zone déplétée. Quand on augmente le courant, la population d'électrons et de trous augmente dans la zone déplétée; de manière correspondante le quasi-niveau de Fermi en bande de conduction (de valence) $E_{f, c}\left(E_{f, v}\right)$ s'élève (s'abaisse) et se rapproche du bas de la bande de conduction (resp. du haut de la bande de valence). La différence entre les deux quasi-niveaux de Fermi, qui sont chacun déterminés par les niveaux de dopage dans les zones $n$ et $p$, est égale à la chute de potentiel $\mathrm{eV}$ : en appliquant une tension $V$ sur la jonction, on a brisé le niveau de Fermi en deux quasi-niveaux de Fermi distants d'une quantité eV au niveau de la zone de charge d'espace, comme cela est illustré sur la figure 7. La probabilité d'occupation en bas de bande de conduction et en haut de bande de valence devient à un certain point supérieure à $1 / 2$, on a alors plus d'émission que d'absorption dans la zone spectrale proche du gap, et on a dépassé ce que l'on appelle le courant de transparence. Le gain est donné par une expression du type :

$$
g(\omega)=\alpha_{0}(\omega)\left\lfloor f_{c}\left(E_{i}\right)-f_{v}\left(E_{f}\right)\right\rfloor,
$$

où $\alpha_{0}(\omega)$ est le coefficient d'absorption pour une température nulle, et $f_{c}\left(E_{i}\right)$ (resp. $f_{v}\left(E_{f}\right)$ ) la probabilité d'occupation d'un état initial (resp. final) dans la bande de conduction (resp. de valence), c'est-à-dire la fonction de Fermi-Dirac écrite avec le quasi-niveau de Fermi $E_{f, c}\left(\right.$ resp. $\left.E_{f, v}\right)$. Dans cette équation, le gain est donc une fonction du nombre de porteurs (et donc du courant d'après (2)) à travers une dépendance du quasi-niveau de Fermi $E_{f, c}$ en $n$ du genre (le même type d'équation existe dans la bande de valence) :

$$
n=\int_{E g}^{+\infty} D_{c}(E) f_{c}\left(E, E_{f, c}\right) \mathrm{d} E,
$$

où $D_{c}(E)$ est la densité d'état dans la bande de conduction. Pour avoir un gain positif (ou, autrement dit, une inversion de population), il faut nécessairement $f_{c}\left(E_{i}\right)-f_{v}\left(E_{f}\right)>0$, ce qui implique finalement, en écrivant les fonctions de Fermi-Dirac :

$$
q V=E_{f, c}-E_{f, v}>h v,
$$

où $h v$ est l'énergie des photons émis. Cette inégalité est appelée condition de Bernard et Duraffourg. Elle indique notamment que si l'on veut réaliser un laser à semiconducteur orange (énergie des photons supérieure à $2 \mathrm{eV}$ ), il faudra nécessairement appliquer une tension supérieure à $2 \mathrm{~V}$ sur la jonction. 
Nous avons vu qu'en augmentant la tension appliquée sur la jonction $p-n$, on peuple de plus en plus la zone de charge d'espace d'électrons et de trous, en même temps que le courant augmente suivant l'équation (2). À un certain point, on atteint enfin l'inversion de population puis le gain nécessaire à l'oscillation laser : c'est le courant de seuil. Mais oublions un moment le gain pour revenir à la cavité laser. Elle est simplement formée de deux faces clivées du semiconducteur, qui jouent le rôle de miroirs. Le clivage suit théoriquement un plan cristallin, ce qui assure une planéité «miroir ». Dans un premier temps, le coefficient de réflexion de Fresnel à l'interface semiconducteur-air suffit à donner un coefficient de réflexion de $30 \%$ environ. Nous verrons que dans les réalisations actuelles, des miroirs multicouches peuvent être déposés sur ces faces clivées pour améliorer les performances du dispositif. Ceci n'est néanmoins pas indispensable à l'obtention de l'effet laser dans un semiconducteur. On touche ici à un point central du dispositif : sa simplicité. Contrairement aux lasers classiques, encombrants, dans lesquels plusieurs éléments optiques doivent être alignés précisément, le laser à semiconducteur est un simple parallélépipède de matériau. L'alignement entre les deux miroirs est assuré par le parallélisme naturel entre deux plans cristallins de clivage.

On atteint le courant de seuil pour l'effet laser quand l'intensité optique ayant parcouru un aller-retour dans la cavité est revenue au même niveau :

$$
I_{0} \times \exp (g L) \exp (-\alpha L) \times R_{1} \times \exp (g L) \exp (-\alpha L) \times R_{2}=I_{0} .
$$

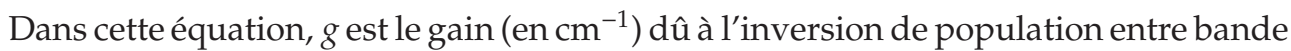
de conduction et bande de valence, et $\alpha$ le coefficient d'absorption du mode optique (due à l'absorption par des porteurs libres ou par d'autres bandes ..., à la diffusion sur des impuretés) entre les deux miroirs $R_{1}$ et $R_{2}$. Ceci mène sans peine à l'équation de seuil :

$$
g=\alpha+\frac{1}{2 L} \ln \left(\frac{1}{R_{1} R_{2}}\right) .
$$

Dans cette équation incontournable, les pertes optiques peuvent être égales à quelques $\mathrm{cm}^{-1}$ ou quelques dizaines de $\mathrm{cm}^{-1}$. Les pertes miroirs valent $40 \mathrm{~cm}^{-1}$ pour une longueur de cavité de $300 \mu \mathrm{m}$ et un coefficient de réflexion de Fresnel de 0,3 . Il faut donc un gain de plusieurs dizaines $\mathrm{de}_{\mathrm{cm}}^{-1}$ pour atteindre le seuil laser. Ce type de gain est considérable si on le compare à un laser à gaz par exemple : c'est ici la densité d'état de la matière condensée qui mène à ce type de gain très élevé, et qui permet finalement de réaliser des dispositifs d'une compacité remarquable (300 $\mu \mathrm{m}$ de long sur quelques $\mu \mathrm{m}$ carré de section). La simplicité, ajoutée à la petite taille et à la capacité de fabrication collective par l'industrie des semiconducteurs, expliquent le prix de revient extrêmement faible de ces dispositifs ( 1 \$ pour les lasers les plus simples dans les lecteurs de disques compacts).

Le schéma du laser à semiconducteur que nous avons exposé jusqu'à présent est assez simple : à un courant $I$ correspond une certaine densité de porteurs dans la zone active (Éq. (2)). De manière équivalente, la tension appliquée correspond à une certaine position des quasi-niveaux de Fermi (en négligeant les résistances séries venant des contacts par exemple). Les deux phrases précédentes sont bien sûr reliées par les 
densités d'états dépendant de la structure de bande du matériau. Une fois connus ces paramètres, on peut donc calculer le gain optique en fonction de la longueur d'onde, connaissant les états électroniques dans les bandes (c'est-à-dire les éléments de matrice optique). Pour savoir si ce courant $I$ a dépassé le seuil d'oscillation laser $I_{\text {th }}$, il suffit de comparer ce gain aux pertes (absorption et pertes optiques) suivant l'équation (3). Avec ce raisonnement, on a l'impression qu'il suffit d'augmenter le courant et qu'on atteindra toujours le seuil laser à un certain point.

En réalité, tout ce que nous avons décrit jusqu'à présent ne suffit pas à faire un bon laser à semiconducteur. Il semble à ce point qu'il ne suffit que d'augmenter le courant pour atteindre le courant de transparence, avoir du gain, et continuer encore à augmenter le courant et la densité de paires électron-trou et donc le gain jusqu'à atteindre le seuil. En réalité, dans les premières structures telles qu'elles étaient réalisées au début des années 60, c'est-à-dire de simples jonctions $p$ - $n$ telles que décrites jusqu'à présent, les courants nécessaires sont monumentaux et responsables d'une telle dissipation thermique dans le composant que le fonctionnement n'est possible qu'à basse température en régime pulsé. Ce qui donna lieu au genre de réflexion suivante dans le monde des télécommunications, qu'il est toujours facile et amusant de ressortir quelques années plus tard :

"Jack Tillman said: "Did you know the semiconductor laser has just been invented ? ... I think we should get some and see if they 're any use at all for communications."

So I went in 1963 to the Electronic Research Laboratory in Baldock, north of London for a month, and brought some of these things with me. They only operated at liquid nitrogen temperature, only operated in pulses of about 10 microseconds, and it took 100 amperes in each pulse to drive the bloody things.

We concluded that they weren't very promising »

David Newman, British Post Office Research Laboratories.

Pour passer d'un dispositif qui marche en pulsé à l'azote liquide à des lasers à semiconducteurs qui fonctionnent de manière stable, en continu, pendant des dizaines de milliers d'heures à température ambiante au fond des océans, plusieurs révolutions ont eu lieu. Schématiquement, deux.

\section{LE LASER À DOUBLE HÉTÉROSTRUCTURE}

La première révolution fut celle du laser à double hétérostructure (on dit « deubeul

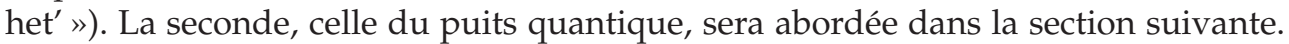
Pour faire du laser à semiconducteur un dispositif efficace, qui marche en continu à température ambiante, il a fallu diminuer le courant de seuil à des valeurs inférieures au niveau où la dégradation thermique commence. Diminuer le courant de seuil implique augmenter le gain pour un même courant. En considérant l'équation (2), on voit que le seul paramètre sur lequel on puisse jouer est la largeur typique de la zone active $d$, où s'accumulent, sous champ, électrons et trous avant recombinaison. Dans une jonction $p$ - $n$ typique, $d$ vaut de l'ordre de $1 \mu \mathrm{m}$. La figure 8 montre la structure de 


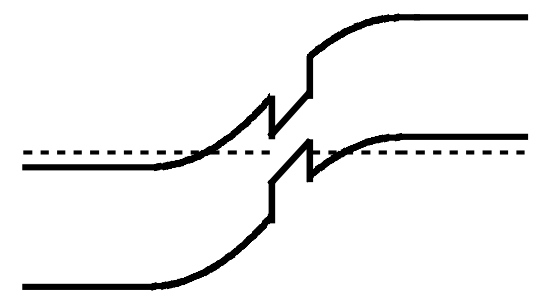

Fig. 8. Double hétérostructure.

bande d'une double hétérostructure : on reconnaît l'allure typique de la jonction $p$ - $n$ avec son champ interne, mais il apparaît en plus un puits de potentiel pour électrons et pour trous, au centre même de la zone de charge d'espace. Ceci est obtenu par l'assemblage de trois couches, deux couches de grand gap de chaque côté d'une couche de bande interdite plus faible.

On ne peut pas assembler n'importe quels semiconducteurs ensemble, il est nécessaire qu'ils aient même paramètre de maille cristalline (comme par exemple GaAs et $\mathrm{AlGaAs}$ ) pour que leur association puisse former globalement un monocristal du point de vue structurel, c'est-à-dire sans trop de dislocations. Si l'on essaye de rassembler deux semiconducteurs quelconques sans qu'il y ait de compatibilité entre leurs paramètres cristallins, $l^{\prime}$ ensemble va présenter énormément de dislocations qui sont autant de chemins non-radiatifs pour les électrons ou les trous. Pour obtenir un bon laser, il est capital d'avoir un bon rendement quantique interne, c'est-à-dire de maintenir autant que possible la validité du schéma de structure de bande (des électrons dans la bande de valence, des trous dans la bande de conduction), sur lequel repose l'inversion de population et qui nécessite l'intégrité de la structure cristalline. Le type de structure représenté schématiquement en figure 8 est réalisé par des techniques de croissance cristallines très sophistiquées d'épitaxie par jets moléculaires ou d'épitaxie en phase vapeur, qui sont parfaitement maîtrisées au niveau industriel. Bien que l'intégrité du cristal soit conservée sur l'ensemble des trois couches de la figure 8 , le monocristal ne présente néammoins pas la même structure de bande dans le matériau central que dans ses deux voisins ; la différence de bande interdite entre les deux résulte en un puits de potentiel dans lequel se rassemblent électrons et trous. La largeur $d$ intervenant dans l'équation (2) est maintenant celle de cette couche, qui peut être arbitrairement petite. En prenant une largeur de typiquement $100 \mathrm{~nm}$, on voit que, dans ce véritable entonnoir à électrons, pour un même courant le nombre de porteurs et donc le gain est augmenté d'un ordre de grandeur, ce qui est considérable.

Un autre avantage tout aussi important a permis à la double hétérostructure de faire de la diode laser un dispositif performant : il s'agit du guidage des photons. Il se trouve en effet que la couche centrale, de plus faible bande interdite, est également de plus fort indice optique. La couche centrale constitue donc un guide plan très efficace pour la lumière. Cela permet de confiner l'onde laser dans la dimension verticale, c'est-à-dire d'éviter que la lumière ne s'échappe et quitte la trajectoire optimale dans l'axe des deux miroirs de la cavité. Comme ces deux miroirs sont plans, il 


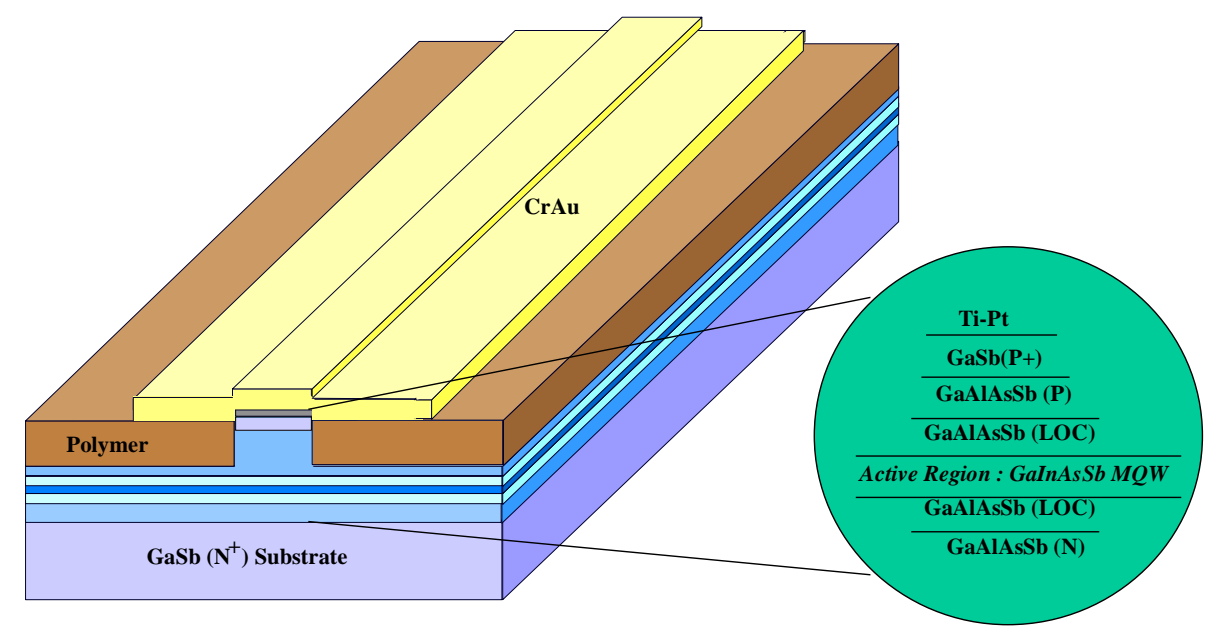

FIG. 9. Figure schématique d'une diode laser après les étapes technologiques.

n'y a en effet pas de stabilité optique a priori et la structure guidante de la figure 8 résoud ce problème dans une dimension, celle perpendiculaire aux couches. Dans la dimension latérale (dans le plan des couches), plusieurs solutions sont également possibles pour guider la lumière. La figure 9 montre un exemple d'une structure laser moderne réalisée actuellement. Outre la multiplicité des couches sur laquelle nous reviendrons, cette figure montre surtout la manière dont on a réalisé un guide d'onde «ruban». La gravure d'un ruban de semiconducteur ("planarisé » ensuite par une couche polymère avant le dépôt de l'électrode métallique sur le dessus) apporte ce guide d'onde latéral qui complète le guide d'onde planaire dans le plan des couches semiconductrices.

La double hétérostructure a permis l'observation d'un effet laser en régime continu et à température ambiante en URSS et aux USA de manière plus ou moins indépendante au printemps 1970. Huit années tout de même après la première observation de l'effet laser à basse température et en régime pulsé, la diode laser commençait à devenir un dispositif réellement intéressant pour les applications. Il allait falloir attendre encore une décennie, les progrès des techniques de croissance des couches, de la technologie, et la deuxième révolution, celle des puits quantiques, pour que les diodes lasers atteignent des performances excellentes en termes de fiabilité, ce qui allait leur ouvrir les portes des télécommunications et du stockage de l'information.

\section{LE LASER À PUITS QUANTIQUES}

Concernant les deux avantages du laser à double hétérostructure (un entonnoir pour les électrons ; un guide pour les photons), on peut se demander quelle est l'épaisseur $d$ optimale pour l'épaisseur de la région active, de plus faible bande interdite. Considérant à nouveau l'équation (2), il semble que pour augmenter la densité de porteurs 


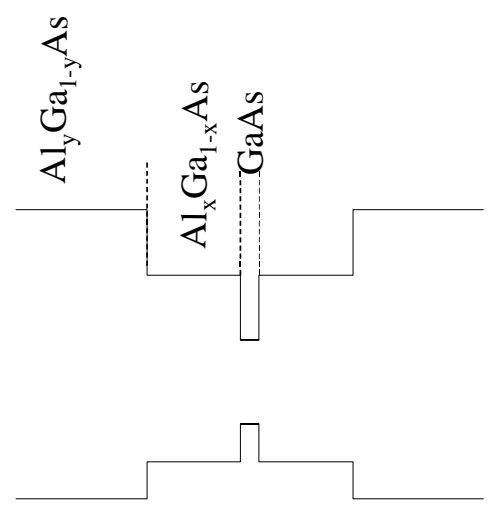

SCH laser

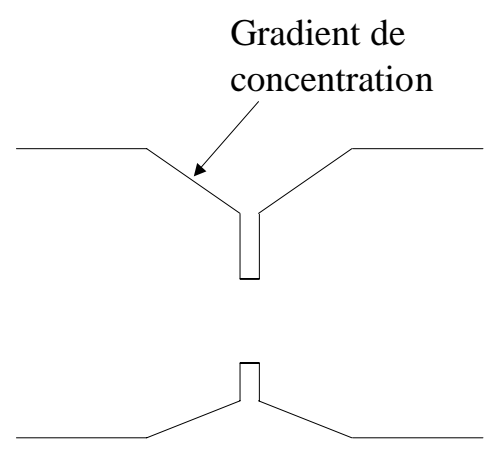

GRINSCH laser

FIG. 10. - Deux exemples de structures laser à confinement séparé (SCH comme Separate Confinement Heterostructure).

pour un courant donné il n'y ait pas de limite inférieure : plus petite est la région active, plus bas sera le seuil du laser. En revanche, il est clair que la capacité d'une couche mince à guider les photons diminue quand l'épaisseur de la couche guidante devient trop faible devant la longueur d'onde optique. Alors qu'une couche de $10 \mathrm{~nm}$ est un parfait entonnoir à électrons, une onde électromagnétique de longueur d'onde $1 \mu \mathrm{m}$ dans le vide est très mal guidée par une telle couche ; le mode électromagnétique guidé s'élargit pour s'évaporer totalement dans les couches voisines. Cette dissymétrie vient du fait que la longueur d'onde des photons est beaucoup plus grande que la longueur d'onde de De Broglie des électrons. Comme la longueur d'onde représente fondamentalement la distance typique en dessous de laquelle le champ électromagnétique refuse de varier de manière trop importante, suivant les équations de Maxwell, il est impossible de comprimer une onde sur une distance plus faible que la longueur d'onde. D'où l'idée d'une structure à confinement séparé, dans laquelle une couche centrale a pour but de confiner les électrons et d'autres couches, plus larges, sont optimisées de manière séparée pour optimiser le guide d'onde optique. Le type de structure à confinement séparé, toujours utilisé dans les diodes lasers actuelles, est représenté sur la figure 10. Le champ interne n'a pas été représenté sur ces schémas, où n'apparaît que la structure de bande plate des matériaux indépendamment de leur dopage. On voit que l'on a affaire à des structures plus complexes et on entre là dans ce que l'on a appelé l'ingénierie de structure de bande. Deux couches de bande interdite et d'indice intermédiaire servent de guide d'onde, leur épaisseur est optimisée pour obtenir un bon guide d'onde, en tenant éventuellement compte de la densité de puissance optique pas trop grande que l'on souhaite pour limiter les effets thermiques dus à la réabsorption. La couche centrale, de gap minimal, peut être maintenant aussi petite que l'on veut pour accroître la densité d'électrons. Dans des structures telles que celle de la figure 10 à gauche, présentant des discontinuités de bande interdite en dehors de la couche active centrale, des électrons et des trous 

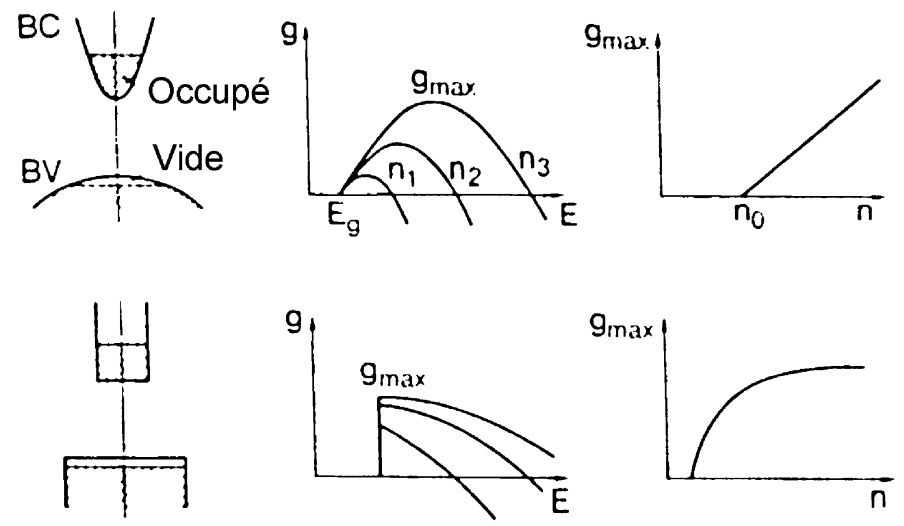

FIG. 11. - Gain dans une double hétérostructure « en volume » ou dans une structure à puits quantiques bidimensionnelle (d'après Weisbuch et Vinter, Quantum Semiconductor Structures, Academic Press).

peuvent éventuellement rester piégés dans des petits puits de potentiel triangulaires formés sous champ par ces discontinuités. De cette remarque résulte la structure à indice graduel (en anglais GRINSCH laser comme GRaded INdex Separate Confinement Heterostructure laser), montrée sur la figure 10 à droite. Pour réaliser ce type de potentiel graduel, c'est la concentration de l'alliage $\mathrm{Al}_{x} \mathrm{Ga}_{1-x}$ As qui varie continûment lors de la croissance. En épitaxie par jets moléculaires par exemple, ceci peut être obtenu en imposant une rampe de température à l'une des sources d'un des éléments de l'alliage dans le bâti d'épitaxie, la variation de température modifiant la proportion d'atomes émis pendant le dépôt et donc incorporés dans la couche épitaxiée. Au-delà de ce type de prouesse, d'une manière générale, les progrès des structures lasers ont très largement reposé sur l'amélioration de la qualité de la croissance des matériaux (pureté, contrôle des dopages, qualité des interfaces...).

La couche centrale très mince (de l'ordre de quelques nanomètres) a maintenant une dimension de l'ordre de la longueur d'onde de De Broglie des électrons. Des effets de confinement quantique apparaissent dans cette couche qui est un puits de potentiel quantique, dans la direction perpendiculaire aux couches. À ce point, la dépendance du gain optique avec le nombre de porteurs change fondamentalement de régime, car il est nécessaire de s'intéresser aux effets particuliers apportés par le confinement quantique. En effet, dans un matériau massif la densité d'état est parabolique alors que dans un puits quantique la densité d'état est constante par morceaux. Ceci est une conséquence de la nature bidimensionnelle du mouvement des électrons dans le plan de la couche quantique, le mouvement dans la troisième direction étant confiné par le puits de potentiel. Imaginons une population d'électrons et de trous peuplant un matériau massif d'une part et un puits quantique d'autre part. Dans le cas massif, on observe un gain en fonction de l'énergie qui croît lentement au dessus du gap, suivant la croissance lente de la densité d'état, et qui redescend ensuite quand on approche du niveau de Fermi pour finalement s'annuler quand il n'y a plus de porteurs (voir Fig. 11). En revanche, dans le cas du puits quantique, 
la croissance du gain au-dessus du gap est beaucoup plus abrupte car la densité d'état est immédiatement maximale dès que l'on a franchi le gap, qui correspond à la différence entre les niveaux fondamentaux en bande de conduction et en bande de valence $E_{1}$ et $H_{1}$. La courbe de gain en fonction de l'énergie, pour un même nombre de porteurs est donc beaucoup plus fine et monte donc beaucoup plus haut, et franchit le seuil plus facilement. Tout ceci est illustré sur la figure 11. Pour cette raison, les puits quantiques ont mené là encore à une forte réduction du seuil des lasers à semiconducteurs. Ces effets de densité d'état sont traités par exemple dans le livre de Weisbuch et Vinter, Quantum Semiconductor Structures (Academic Press). Notons que si l'on cherche à calculer complètement le gain dans un puits quantique pour une certaine population de porteurs et une certaine température, on doit résoudre un calcul des énergies et états propres dans des puits quantiques, prenant en compte les différentes sous-bandes de valence et la bande de conduction. Ces problèmes un peu techniques dépassent de très loin le cadre de cette introduction aux diodes lasers, et le lecteur est invité à consulter par exemple la thèse d'Arnaud Fily (Modèles pour diodes laser de puissance, Paris XI, 1998) pour de plus amples informations. Les interactions coulombiennes peuvent également affecter énormément le gain optique dans les hétérostructures, on consultera par exemple l'ouvrage de Chow et Koch, Semiconductor Laser Fundamentals (Springer).

\section{DES COMPOSANTS DE PUISSANCE}

Nous avons beaucoup évoqué le seuil d'une diode laser et les différents moyens de diminuer ce seuil, ce qui a constitué la principale préoccupation de la communauté travaillant sur les lasers à semiconducteurs pendant une bonne vingtaine d'années. Désormais les seuils des lasers à semiconducteurs sont assez bas (typiquement 200 A. $\mathrm{cm}^{-2}$ ) mais d'autres performances font l'objet d'une recherche sans répit. Parmi celles-ci, la puissance maximale que peut sortir une diode laser.

Quand on cherche à sortir le maximum de puissance d'une diode laser, on augmente le courant et la puissance de sortie augmente presque linéairement avec ce courant au-dessus du seuil. Comme on l'a vu, le seuil est déterminé par les pertes par absorption (pertes internes) et les pertes optiques (vers l'extérieur de la cavité, qui sont tout de même des pertes utiles). Au-dessus du seuil, augmenter le courant revient encore à augmenter le nombre de porteurs qui traversent la zone active (par définition même du courant). Pourtant, le gain ne peut pas physiquement dépasser sa valeur au seuil donné par l'expression (3) : sinon on aurait une onde optique croissante dans la cavité, ce qui ne correspond pas à une solution physique stationnaire. L'égalité (3) donne donc le gain au seuil, mais aussi au-dessus du seuil, où le gain reste alors constant. Cela implique que le nombre de porteurs $n$ est constant, ce qui n'est compatible avec l'augmentation du courant que si leur temps de vie moyen diminue. C'est exactement ce qui se passe grâce au phénomène d'émission stimulée. Au-dessus du seuil, les porteurs incidents dans la zone active sont convertis en photons par émission stimulée, de manière beaucoup plus rapide que sous le seuil ; c'est ce qui permet de conserver leur population constante quand le courant augmente. Autrement dit, tout courant au-dessus du seuil est converti en lumière, mis à part 


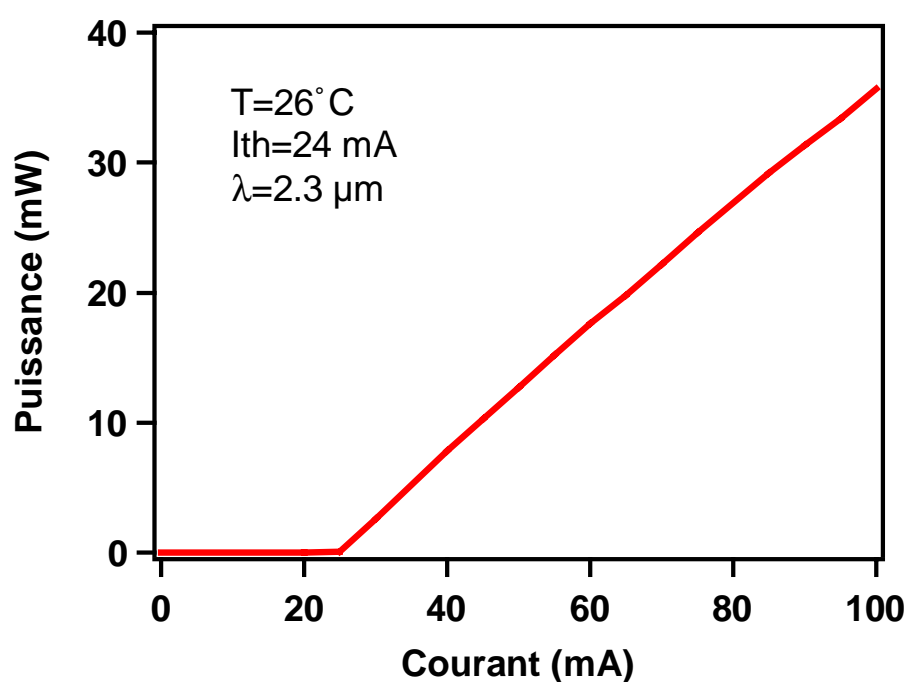

Fig. 12. - Caractéristique $I(V)$ d'une diode laser. Il s'agit ici d'une diode laser à base d'Antimoine (le matériau composant les puits quantiques sont dans un alliage quaternaire GaI$\mathrm{nAsSb}$ ), émettant dans le moyen infrarouge (collaboration Université de Montpellier/Thomson CSF LCR). Le courant de seuil est de $24 \mathrm{~mA}$.

certaines pertes toujours présentes (absorption des photons émis, recombinaisons non radiatives) qui permettent de définir un rendement différentiel en watt/ampère. Il s'agit de la pente de la caractéristique $I(V)$ de la diode laser, dont un exemple est montré sur la figure 12.

À très haut courant d'injection, les caractéristiques de la diode finissent néanmoins par se dégrader, parfois définitivement. La dégradation des performances peut provenir d'abord de l'augmentation des canaux non-radiatifs avec le courant (courants de fuite, mécanismes de recombinaison non radiative plus efficaces pour une densité de porteurs élevée...). Il peut exister également des problèmes thermiques, les mêmes problèmes qui ont rendu les progrès des lasers à semiconducteurs assez lents au début de leur histoire. Les pertes non radiatives (en particulier sur les surfaces du cristal, au niveau des facettes miroirs), l'absorption résiduelle contribuent à chauffer le composant. Plusieurs phénomènes s'associent pour une augmentation de la température : quand la température augmente, le gain optique diminue, ainsi que le gap du matériau. Tout ceci contribue à accroître l'absorption et donc la température. La montée en température de la structure peut aussi contribuer aux migrations de dislocations qui vont augmenter les pertes non radiatives et donc l'échauffement : on se trouve en présence d'une spirale de dégradation catastrophique. Par exemple, sur les composants de puissance GaAs/AlAs, au niveau des facettes, les pertes non radiatives sont plus importantes en raison d'états de surface spécifiques favorisant les recombinaisons non radiatives. La structure commence alors à se dégrader à haute puissance pour tuer finalement le composant. Pour traiter ces problèmes, le 


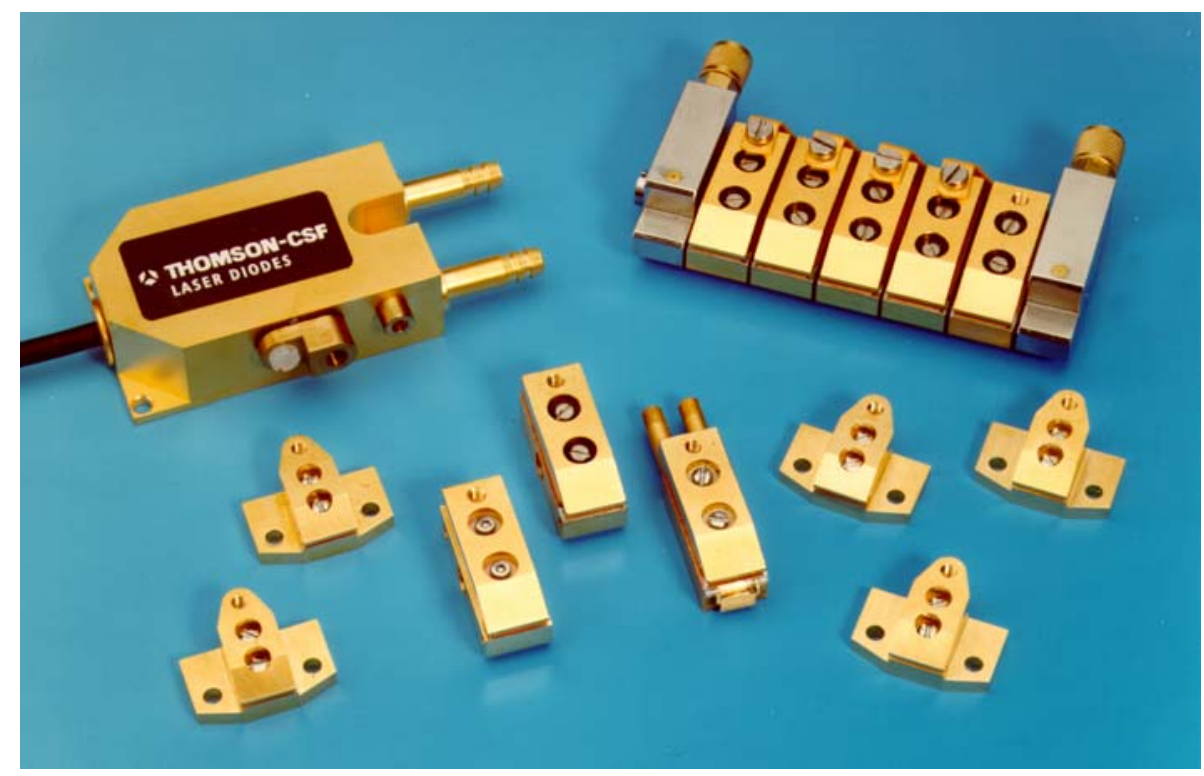

FIG. 13. - Exemple de produits «barettes de puissance» vendus par Thomson-CSF Laser Diodes.

traitement des surfaces (passivation, dépôts de miroirs diélectriques qui ont également une fonction de limitation de la fragilité des facettes vis-à-vis du flux optique) ainsi que l'évacuation de la chaleur par l'embase sur laquelle est soudée le semiconducteur sont des points très délicats nécessitant un important savoir-faire.

Pour obtenir toujours plus de puissance, il est naturel d'assembler différents lasers côte à côte sur une barrette laser (au détriment naturellement de la qualité de faisceau globale). La gestion de la dissipation thermique de ces barrettes est un point crucial, d'autant plus difficile à résoudre que la densité de composants est grande. On peut avoir éventuellement recours à une circulation de fluide dans les embases en cuivre pour refroidir les composants (voir Fig. 13). Si l'on veut toujours plus de puissance, on peut empiler des barrettes ensemble. Une barrette de $1 \mathrm{~cm}$ de large peut sortir aujourd'hui 50 watts continu ou 100 watts quasi continu (c'est-à-dire avec un taux de remplissage de quelques \%); un empilement de barrettes peut fournir des puissances supérieures à 300 watts continu et des puissances crêtes focalisées dépassant les $10 \mathrm{~kW} \cdot \mathrm{cm}^{-2}$.

\section{QUELQUES STRUCTURES LASER PARTICULIÈRES}

Nous n'avons évoqué jusqu'à présent que des structures laser standard. Un grand nombre de variations ont été proposées dans la littérature. Nous évoquons rapidement deux d'entre elles, en raison de leur importance au niveau des applications.

La première est le laser à contre-réaction distribuée. Pour les applications en 
télécommunications à longue distance, il est capital de disposer de sources d'une pureté spectrale très élevée. En effet, la dispersion dans les fibres optiques est responsable d'une différence entre les vitesses de propagation de fréquences différentes, résultant en un décalage sensible sur de longues distances, même pour des fréquences très proches. C'est finalement ce phénomène qui limite la bande passante des télécommunications fibrées. Le laser à semiconducteur présentant un gain large (si on le compare aux lasers à gaz ou aux lasers $\mathrm{Nd}^{3+}$ :YAG par exemple), il n'est pas un laser très monochromatique $a$ priori et il présente une multitude de modes longitudinaux dans son spectre de sortie. Le laser à contre-réaction distribuée (ou laser DFB pour Distributive FeedBack) permet d'obtenir un spectre de sortie monofréquence. Un réseau périodique est gravé sur la région active du laser, de période égale à une demi-longueur d'onde du laser. On sait que la propriété d'un réseau d'indice périodique est de présenter un coefficient de réflexion dépendant fortement de la longueur d'onde, avec en particulier une résonance quand la période du réseau est égale une demi-longueur d'onde. Les propriétés de rétroaction du faisceau laser à l'intérieur du guide d'onde dépendent donc énormément de la longueur d'onde autour de cette résonance. Il en résulte une compétition très inégale entre les différents modes longitudinaux qui aboutit à la sélection d'un seul d'entre eux, ce qui est l'effet recherché. L'ouvrage de Yariv Quantum Electronics expose les principes fondamentaux de ces structures optiques très intéressantes. On pourra aussi consulter l'ouvrage plus spécialisé Distributed Feedback Semiconductor Lasers de Caroll et al., édité par la SPIE.

Si l'on désire un laser d'une pureté spectrale encore supérieure, il est possible d'utiliser une géométrie en cavité externe : dans ce cas, un miroir antireflet est déposé sur la face de sortie de la diode laser et le rayonnement est réfléchi puis réinjecté dans le laser à l'aide d'un réseau de diffraction par exemple. La cavité, maintenant beaucoup plus longue, présente des modes longitudinaux très fins. La compétition entre ces modes est extrêmement efficace en raison de la très grande sensibilité en longueur d'onde de la rétroaction.

Les lasers verticaux émettant par la surface sont un autre type de structure utilisant également les propriétés réfléchissantes des structures d'indice périodique. Le but est de réaliser un laser émettant non plus dans le plan des couches, mais perpendiculairement à la surface des couches semiconductrices. L'intérêt est naturellement la densité encore plus grande de lasers qu'il est alors possible de réaliser par unité de surface d'un substrat : la grande dimension d'un laser standard est en effet sa longueur dans le plan des couches. Pour obtenir une émission par la surface, il est nécessaire de réaliser une cavité ; mais cette fois les faces planes du semiconducteur ne suffisent plus. Considérant l'équation de seuil (3), on voit que si la longueur de la cavité $L$ est réduite de $300 \mu \mathrm{m}$ à quelques microns (épaisseur typique de croissance des semiconducteurs), le seuil va augmenter de manière prohibitive si l'on ne dispose pas de très bons miroirs. En pratique, il est nécessaire d'avoir des miroirs de coefficient de réflexion supérieur à $99 \%$ de part et d'autre de la couche active. Ces miroirs sont obtenus par croissance ou par dépôt de couches d'indices différents. On réalise ainsi une structure d'indice périodique, appelée miroir de Bragg, en raison de l'analogie entre la réflexion de la lumière par une structure périodique par interférences constructives entre les réflexions sur les différentes interfaces et la réflexion des rayons X par les plans d'atomes périodiques dans les structures cristallines. La diode laser finale est donc la succession d'un premier miroir de Bragg, de la cavité 
qui comprend la région active avec un ou plusieurs puits quantiques, et d'un second miroir de Bragg qui referme la cavité. L'un des deux miroirs est moins efficace car il comporte moins de couches que l'autre, de telle sorte que la lumière sort essentiellement d'un seul côté. Ces structures émettant par la surface ont été décrites dans l'article de Jewell et al., IEEE J. Quant. Electron. 27 (1991) 1332; des réalisations plus récentes sont décrites dans Vertical Cavity Surface Emitting Lasers III, édité par Kent Choquette et Chun Lei (SPIE Vol. 3627).

Il existe d'autres composants particuliers que nous n'avons pas évoqués ici, citons par exemple les lasers à boites quantiques (voir Bimberg et al., Quantum dot heterostructures, John Wiley \& Sons, 1999), les lasers à modes bloqués (voir par exemple Chen et Wu, IEEE J. Quant. Electr. 28 (1992) 2176), ou encore les lasers pompés optiquement (voir par exemple Le et al., Appl. Phys. Lett. 63 (1993) 1465).

\section{SYNTHÈSE}

Tentons de résumer en quatre points les grandes directions de recherche qui ont permis aux lasers à semiconducteurs d'être les dispositifs exceptionnels que l'on connaît aujourd'hui :

- l'optimisation du gain par électron transporté : d'abord dans une double hétérostructure, puis dans un puits quantique, voire un puits quantique contraint, la contrainte permettant de modifier les structures de bandes dans le puits quantique pour optimiser la densité d'état jointe vers un meilleur gain optique ;

- l'optimisation du guide d'onde de la lumière : dans une double hétérostructure encore ou dans des structures à confinement séparé, mais aussi grâce au guide d'onde latéral réalisé par diverses méthodes technologiques ;

- la qualité du matériau et l'amélioration constante des techniques de croissance : pour obtenir des matériaux sans défauts, pour éviter les pertes non radiatives. La comparaison entre les différents matériaux possibles peut être intéressante. Par exemple, des études ont montré que les matériaux sans aluminium présentaient une meilleure résistance à la dégradation catastrophique des lasers à haute puissance. Ce genre de thème mobilise aujourd'hui beaucoup de chercheurs;

- la qualité de la technologie : réalisation des guides d'onde latéraux gravés, des contacts ohmiques de bonne qualité entre les contacts métalliques et les couches semiconductrices dopées pour éviter toute résistance série, source de dissipation thermique néfaste. La qualité des clivages et le dépôt de miroirs multicouches optimisés sont également tout un art, déterminant les taux de recombinaison de surface et l'amplitude de la réabsorption du flux laser sur la facette. Enfin, la brasure des structures sur un bon dissipateur thermique conditionne énormément la température de la jonction du laser en fonctionnement, et donc finalement les puissances que les lasers peuvent sortir.

Ces quatre grands thèmes (structure électronique, structure optique, matériau, technologie) sont toujours aujourd'hui autant de domaines de recherche pour une communauté très large. Le lecteur intéressé par les dernières évolutions en matière de 

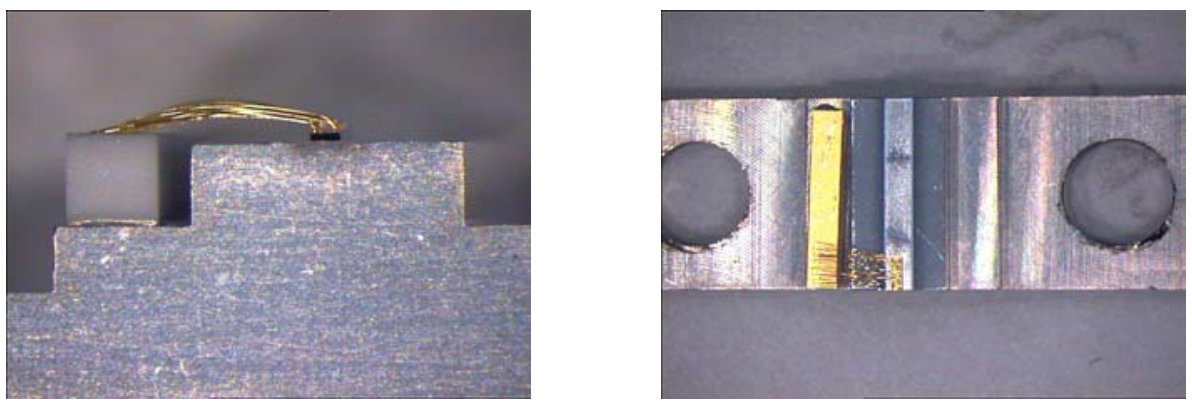

FIg. 14. Diode laser montée sur son embase.

recherche sur les lasers à semiconducteurs pourra par exemple consulter les comptesrendus des conférences Photonic West, CLEO, ou LEOS.

La photographie de la figure 14 montre un composant typique actuel. La photographie à gauche montre la face du dispositif. On voit en fait essentiellement l'embase en cuivre qui sert de dissipateur thermique (et de contact métallique sous la diode), ainsi que les multiples fils d'or thermocompressés sur le dessus du dispositif, qui amènent le courant dans la diode. Ces fils sont rapportés sur une pièce en céramique sur la gauche de la photographie, sur laquelle il est facile de souder un fil électrique standard. La figure de droite est une vue de dessus. Les deux trous à droite et à gauche servent simplement à fixer l'embase du laser quelque part à l'aide de deux vis; on distingue à gauche la céramique pour le report de contact et les fils d'or. On devine, en bas au centre, la puce semiconductrice, d'une taille très faible devant son embase en cuivre (300 $\mu \mathrm{m}$ de long typiquement).

\section{CONCLUSION}

Les quelques chiffres suivants laissent rêveurs quant au succès technique et commercial des lasers à semiconducteurs : un chiffre d'affaire total de 3 Milliards de $\$$ en 1999 et une croissance record de $47 \%$ dans l'année. Cette croissance s'explique essentiellement par les $70 \%$ pour les applications en télécommunication, considérant la croissance actuelle d'Internet de 300\% par an. Les besoins considérables en matière de télécommunication laissent présager une croissance importante et durable. Le prix moyen d'une diode laser est de 1000 \$ pour les applications télécom (mais la dispersion de prix est importante selon les performances); 1 à 10 \$ pour le stockage optique ; et de 100 \$ le watt typiquement pour les barettes de puissance. La puissance record pour une diode laser (un seul ruban) est de 10 watts typiquement, une barette de $1 \mathrm{~cm}$ de large a sorti en l'an 2000267 watts CW à $300 \mathrm{~K}$, ceci pour une longueur d'onde de $980 \mathrm{~nm}$.

Les lasers à semiconducteurs occupent environ $70 \%$ du marché total des lasers : c'est donc qu'ils présentent des avantages importants mais aussi des limitations qui les empêchent de s'emparer de sa totalité. Leurs spécificités peuvent être simplement résumées de la manière suivante : 
- la densité d'atomes actifs est celle de la matière condensée, et pas celle d'atomes dopants, de liquides ou de gaz. Cette densité mène à des gains gigantesques et donc à des dispositifs compacts (et en particulier intrinsèquement bien adaptés aux applications grand public);

- le mécanisme de conversion électron-photon est très efficace, en raison de la maîtrise stupéfiante de la fabrication d'hétérostructures semiconductrices sur mesure d'une grande pureté, exempte de défauts. En conséquence, les rendements « à la prise » sont très élevés ; ils peuvent atteindre jusqu'à 70\%, c'est-à-dire qu'en consommant 1 watt électrique, il sort de la diode laser 700 $\mathrm{mW}$ de lumière sur un faisceau cohérent;

- la fabrication par nature collective des dispositifs (conséquence également de leur compacité) par l'industrie des semiconducteurs mène à des coûts de production faibles, en spirale vertueuse avec le développement à grande échelle du marché ;

- les différents matériaux accessibles et l'ingénierie de structure de bande permettent de réaliser des lasers à des longueurs d'onde sur mesure dans une certaine gamme (contrairement aux lasers à gaz ou à ions, où les longueurs d'onde sont la plupart du temps figées), voire même faiblement accordable avec la température du laser. On dispose ainsi sur le marché de lasers à semiconducteurs où on le souhaite entre le rouge et l'infrarouge jusqu'à $2 \mu \mathrm{m}$, et même dans le bleu ou l'infrarouge moyen avec des performances beaucoup plus modestes que dans le proche infrarouge.

En ce qui concerne leurs limitations, n'oublions pas que les lasers à semiconducteurs sont des lasers de faible énergie, si on les compare aux lasers solides pulsés par exemple. Cette faible énergie s'explique naturellement par la compacité du dispositif et la puissance limitée qu'il peut supporter dans un volume si faible d'une part, et d'autre part par un temps de vie radiatif court et donc une incapacité à stocker de l'énergie. Le temps de vie de l'ordre de la nanoseconde dans un semiconducteur typique est en effet à comparer à un temps de vie de $240 \mu$ s pour les ions $\mathrm{Nd}^{3+}$ dans la matrice YAG. Les autres points faibles des lasers à semiconducteurs sont d'une part la qualité de faisceau médiocre (comparée à celle des lasers à solides ou à gaz), et d'autre part les domaines de longueur d'onde encore peu ou pas couverts par les diodes lasers : citons par exemple en premier lieu le vert, le bleu ou l'UV, domaines spectraux où les performances des lasers semiconducteurs sont encore faibles (surtout comparées à celles des YAG doublés en fréquence, des lasers Argon ou des lasers à excimères), mais aussi l'infrarouge moyen, où les lasers à cascade quantique offrent des performances intéressantes, mais encore loin de celles des lasers à dioxide de Carbone.

\section{PERSPECTIVES}

Les diodes lasers sont encore loin d'avoir démontré toute l'étendue de leurs possibilités. Une communauté considérable mène une recherche dans laquelle on peut distinguer plusieurs azimuths :

- la conquête de nouvelles gammes spectrales : le bleu, par exemple, fait l'objet d'une recherche très intense pour les applications de stockage de l'informa- 
tion (la plus courte longueur d'onde permet d'accroître la densité de stockage), d'impression laser, ou d'imagerie laser. Cette recherche est difficile car l'émission dans le bleu suppose l'utilisation de semiconducteurs à grands gaps (tels que GaN, voir par exemple l'ouvrage de Nakamura et al., The blue laser diode, Springer, 2ème édition, 2000), moins maîtrisés que les semiconducteurs déposés sur GaAs ou InP. De l'autre côté du spectre, dans le moyen-infrarouge, les matériaux à base d'Antimoine présentent des performances en forte croissance pour des longueurs d'onde situées entre 2 et $3 \mu \mathrm{m}$. Au-delà, les lasers à cascade quantique apparus récemment offrent une gamme très étendue de longueurs d'onde accessibles, entre 4 et plus de $15 \mu \mathrm{m}$. Ces lasers, que nous n'avons pas évoqués ici, reposent non plus sur l'émission bipolaire entre un électron et un trou dans les bandes de conduction et de valence, mais mettent en jeu des transitions entre niveaux électroniques confinés quantiquement dans la seule bande de conduction. Ils sont donc fondamentalement très différents des lasers classiques, même si les ingrédients qui ont fait le succès des lasers à semiconducteurs (maîtrise de la croissance des hétérostructures semiconductrices et de leur technologie) sont là aussi indispensables;

- plus de puissance, plus de brillance : pour les applications de pompage optique des lasers solides tels que le $\mathrm{Nd}^{3+}$ YAG par exemple, pour les applications de pompage optique des amplificateurs ou des lasers à fibre, ou encore pour le traitement des matériaux (découpe, soudure, marquage...);

- des sources monochromatiques (DFB), à des longueurs d'ondes choisies et accordables, pour les applications en télécommunication (multiplexage en longueur d'onde) ; mais aussi en détection de gaz;

- des sources lasers «ultimes" : toute une communauté s'intéresse à des réalisations exotiques, intéressantes dans le cadre de l'optique quantique : sources lasers sans seuil (utilisant des microcavités ou des matériaux à bande interdite photonique), sources de lumière comprimée, sources à photons uniques, sources pour la cryptographie quantique. Le lecteur intéressé est invité à consulter par exemple les compte-rendus de deux écoles d'été s'étant déroulées à Cargèse en août 1995 et 1998 : Microcavities and Photonic Bandgaps : Physics and Applications (NATO ASI Series, édité par J. Rarity et C. Weisbuch, 1996) et Confined Photon Systems - Fundamentals and Applications (Springer, édité par H. Benisty, J. M. Gérard, R. Houdré, J. Rarity et C. Weisbuch, 1999).

\section{Remerciements}

Au cours de l'écriture de cet article, j'ai naturellement profité d'un grand nombre d'interactions avec des spécialistes des lasers à semiconducteurs travaillant au Laboratoire Central de Recherches ou ailleurs. Il serait trop long et trop périlleux de citer toutes ces interactions. Néanmoins, parmi ces personnes, je tiens à remercier tout particulièrement Philippe Collot, Geneviève Glastre, Michel Krakowski et Carlo Sirtori. 